\title{
The Effects of 2-Week 16:8-Hour Intermittent Fasting on Body Composition: Case Study
}

\author{
Pietro Weaver, Alexander Zeizinger, Peter Vu, \\ Hayden West, \& Soon-Mi Choi \\ Midwestern State University, Texas, USA
}

\begin{abstract}
Intermittent fasting is a form of nutrition dieting in which an individual is allotted an amount of time to consume food throughout the day. Intermittent fasting has been shown to acutely change an individual's body composition as an effective intervention. The purpose of this research was to determine the effects of 2-week 16:8-hour intermittent fasting on body composition.
\end{abstract}

Keywords: intermittent fasting, body composition, weight loss

\begin{tabular}{lr}
\hline Article History & Corresponding Author \\
Received 20 September 2020 & Pietro Weaver \\
Accepted 10 October 2020 & pietroweaver@gmail.com \\
Published 31 January 2021 & Department of Kinesiology \\
Available online 19 February 2021 & College of Health Sciences \& Human Services \\
https://doi.org/10.47544/johsk.2021.2.1.31 & Midwestern State University, Texas, USA \\
\hline
\end{tabular}

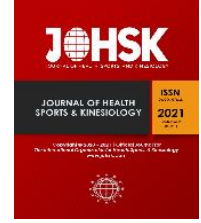

\section{Methods}

Participants ages ranged from 21-24 years, with a total of four (males: 3, female: 1), all college aged students. All participants were involved in regular exercise but had no previous experience of intermittent fasting. Each participant consumed their normal dietary intake till day of pre-intervention testing but were told to be in a fasted state before undergoing baseline testing. A glucometer was used to evaluate fasted blood glucose. Blood pressure was determined by using a manual cuff. A stadiometer was used to measure participants height and weight. A flexible tape measure was used to determine the waist and hip circumferences, which were used to determine the waist to hip ratio. Bioelectrical Impedance Analysis and Skinfold Calipers were used to determine body composition. Participants maintained normal physical activity levels, and logged volume and intensities in the MyFitnessPal application. Participants followed an isocaloric intake. Participants followed a 16:8 ratio, with a 16 hour fast and 8 hours allotted to consume food. Participants followed the intermittent fasting diet for a total of two weeks. Postintervention was reevaluated at the two-week mark following intervention start. Data was presented as mean \pm standard deviation and determined by descriptive analysis. Statistical significance was set at $p<.05$.

\section{Journal of Health, Sports, \& Kinesiology | ISSN 2692-9864 | www.johsk.com}


| 2021 | Volume 2 | Issue 1 | The Journal of Health, Sports, and Kinesiology |

\section{Results}

There was no significant difference in body composition and fasting blood glucose. Significant difference was noted in both body mass and blood pressure.

\section{Conclusion}

The results may indicate that intermittent fasting with a 16:8 ratio can decrease body mass and resting blood pressure in young individuals. Due to COVID-19, limitations were placed allotting only 2 weeks for intervention and for smaller sample size. For future research, study would be increased to 8 weeks with an increased sample size up.

\section{Journal of Health, Sports, \& Kinesiology | ISSN 2692-9864 | www.johsk.com}

